\title{
Role of Exercise as a Therapeutic Intervention for Hypertension
}

\author{
Md. Shahidur Rahman ${ }^{1}$, A.K.M. Salek ${ }^{1}$ \\ ${ }^{1}$ Department of Physical Medicine and Rehabilitation Bangabandhu Sheikh Mujib Medical University \\ Address for Communication \\ Dr. Md. Shahidur Rahman Associate Professor Department of Physical Medicine and Rehabilitation \\ Bangabandhu Sheikh Mujib Medical University, Dhaka \\ E-mail : shahidurphysmed@yahoo.com
}

\begin{abstract} tic intervention for the control of hypertension.

Introduction

Increasing levels of physical activity, together with reduced sodium intake, weight loss, and limited alcohol consumption are therapeutic lifestyle changes known to effectively reduce blood pressure (1). Data from the Coronary Artery Risk Development in Young Adults (CARDIA) study support the fact that cardiovascular fitness is inversely related to the development of hypertension, diabetes, the metabolic syndrome, and hypercholesterolemia (2). Dynamic aerobic training, in particular, has been shown to lower conventional and daytime blood pressure readings among hypertensive patients (3). In general, exercise provides a substantial benefit for patients with both hypertension and cardiovascular disease (CVD) and reduces the 10-year cardiovascular risk by $25 \%$ (4). Two other advantages of exercise as a therapeutic intervention are its positive effect on multiple cardiovascular disease risk factors. Mild to moderate exercise has low risk and very few contraindications for most people.
\end{abstract}

Physical exercise is found to have positive health and well being of a person since time immemorial. Regular exercise has proved to normalize the elevated blood pressure of mild to moderate intensity by many studies. The blood pressure response to such exercise may also be used to screen normotensive persons for the risk of developing elevated blood pressure. For mild hypertension aerobic exercise and life style changes such as weight loss, dietary changes such as salt restriction and cessation of smoking are probably better choices. In moderate to severe essential hypertension, exercise should be combined with life style changes and medication. The aim of this review study is to delineate the effect of exercise in the control of elevated blood pressure. Methods used were analysis of the published article collected from the internet and other sources. The specific aims were to emphasis the importance of exercise as therapeu-

\section{Blood Pressure Response to Exercise}

Exercise has been shown to have both an acute and a chronic effect on blood pressure (BP). The typical blood pressure response to an acute bout of aerobic exercise is a gradual increase in systolic blood pressure (SBP) and a gradual decrease or no change in diastolic blood pressure (DBP). Systolic and diastolic pressures at rest average around 125 and $80 \mathrm{mmHg}$, respectively, with a mean pressure of about $95 \mathrm{mmHg}$.

Therapeutic Intervention for Hypertension brought about by changes in the autonomic nervous system and hormonal influences. This affects systolic pressure more than diastolic or means pressure. The reason for this is that during exercise there is a simultaneous decrease in resistance due to vasodilatation of the arterioles supplying blood to the more metabolically active skeletal muscles. This means that more blood will drain from the arteries through the arterioles and into the active muscle capillaries, thus minimizing changes in diastolic pressure. In turn, changes in mean arterial pressure will also be minimized. These changes in resistance during exercise are impressive. The blood pressure response to exercise differs between normotensive and hypertensive people. In normotensive individuals, acute bouts of dynamic exercise result in a higher SBP (usually $<220 \mathrm{mmHg}$ ) and a reduction in peripheral vascular resistance as described above. However, because of an elevated baseline level, the absolute level of SBP attained during dynamic exercise is usually higher in persons with hypertension $(220 \mathrm{mmHg}$ or more). In addition, DBP may not change, or may even slightly rise, during dynamic exercise, probably as a result of impaired endothelial function and increased sympathetic outflow. Patients with hypertension exhibit an increase in the total peripheral resistance and increases in myocardial oxygen consumption and impaired vasodilation (5). Immediately following an acute bout of exercise, a post exercise hypotension phenomenon has been recognized in both normotensive and hypertensive individuals.

This response may last up to $22 \mathrm{~h}$ after exercise and, due to the decrease in systemic vascular resistance, usually drops the arterial blood pressure by 5-7 $\mathrm{mmHg}$. The reduction in blood pressure after exercise can be sustained for up to 1-2 weeks, after three or more separate bouts of exercise $(6)$. Over time, these changes chronically translate into eccentric left ventricular hypertrophy with a preserved cardiac function (7).

Stretching caused by the overload from exercise translates into cardiomyocyte expansion, hypertrophic remodeling, and an increase in the contractile force per cell (8). This homogenous, physiologic remodeling is beneficial, as opposed to the pathologic concentric hypertrophy related to chronic hypertension, which involves apoptotic processes and the replacement of necrotic tissue by fibroblasts and collagen ${ }^{(9)}$. 
A reduction in the cardiac output and/or a reduction in the peripheral vascular resistance are postulated as possible mechanisms for the antihypertensive effects of exercise training (10-12). Aerobic exercise is also associated with a decrease in plasma noradrenaline, which correlates with the reductions in SBPs and DBPs that are common among hypertensive individuals with elevated resting catecholamine levels.

This reduction in levels of sympathetic activity may correlate with the reduction in cardiac output as well as in peripheral resistance (13). At the endothelial level, the elevated shear stress in the vessel walls, a product of the increased blood flow to muscles during exercise, serves as a stimulus for nitric oxide release, thus leading to smooth musclemediated vasodilatation (14). Reactive hyperemia, an index of the nitric oxide-related vasodilatation, improved with long-term aerobic exercise in a study of Japanese patients with mild-to-moderate essential hypertension (15).

There is also a depletion of whole blood and plasma volumes, as well as sodium depletion, noted by a significant correlation between the changes in the sodium/potassium ratio and SBP (16). These changes were confirmed in a study where middle-aged men with essential hypertension subjected to regular exercise experienced a significant reduction in blood pressure, a higher serum $\mathrm{Na}$ and $\mathrm{K}$ ratio, a higher cardiac index, and lower total peripheral resistance (17). These findings help explain the theory that exercise reduces blood pressure more effectively in volume-dependent hypertensive.

\section{Clinical Evidence}

Data from the First National Health and Nutrition Examination Survey (NHANES I) with 9791 study subjects revealed a favorable association between exercise and outcomes (all-cause and cardiovascular mortality) in hypertensive individuals ( $\mathrm{p}<0.001$ and $\mathrm{p}<0.002$, respectively), when compared to normotensive and prehypertensive subjects (18). The chronic effects of regular exercise on individuals with hypertension are reflected by an average blood pressure reduction of $2.6-7.4 \mathrm{mmHg}$ (19). Thus, prescribing exercise should be a standard of practice, especially in patients with hypertension.

Data from multiple randomized controlled clinical trials support the use of exercise for blood pressure reduction (20). Despite this overwhelming evidence, some aspects remain undefined; one example is whether the reduction in blood pressure is independent of weight reduction, body composition, and other modifiable risk factors. A meta-analysis of 44 randomized controlled clinical trials indicates that endurance exercise contributes to the control of blood pressure in overweight as well as in lean subjects, although a significant decrease in the BMI occurred in the obese population only (21). Although exercise seems to exert its benefit independently from other modifiable factors, blood pressure control was most successful among individuals undergoing multiple behavioral interventions including physical activity, weight loss, decreased sodium intake, decreased daily intake of alcohol, and a diet high in fruits and vegetables and low in saturated and total fat (DASH diet) (22). Another meta-analysis of 54 randomized controlled trials (2419 patients) evaluating the effect of aerobic exercise on blood pressure reported an overall significant reduction in mean SBP 3 to $8 \mathrm{mmHg}$ and mean DBP 2 to $6 \mathrm{mmHg}$ (23). BP was significantly reduced even in trials whose participants did not lose weight. Other factors such as daily emotional fluctuations, stress, and degree of physical activity have also been implicated in the severity of hypertension and its response to exercise. Regular aerobic exercise together with weight loss has particularly proven to be beneficial in the reduction of diastolic and mean arterial blood pressure during activities of daily living. In a study of 112 participants, the elevations of DBP and SBP, associated with the emotional distress and physical activity variations during a typical day, notably improved with 55 min of daily exercise ( $p$ $<0.005)^{(24) \text {. }}$

\section{Exercise and Hypertension in the Elderly}

Progressive degeneration of the arterial media and increased collagen and calcium deposition results in stiffening of the arteries in the elderly, hypertensive population (25). These changes seem to be hastened by the effect of prolonged high blood pressure levels. Clinically, this is manifested by an elevated SBP and has been implicated as a contributor to cardiovascular morbidity and mortality (26). However, moderate aerobic exercise training has failed to improve largeartery stiffness in patients with isolated systolic hypertension (27). Nonetheless, a study involving elderly individuals with essential hypertension that were randomized to a lowor moderate-intensity training program found that lowintensity exercise significantly reduces the systolic BP $7 \mathrm{mmHg}$ after 9 months of regular training $(\mathrm{p}<0.05)$. Both low- and moderate intensity exercise, however, reduced the DBP and mean BP by $9 \mathrm{mmHg}$ and $8 \mathrm{mmHg}$, respectively, when compared with the control group of patients not exercising (28). An increase in the total body fat, particularly abdominal fat that is more prominent with aging, is also directly related to the appearance of hypertension in this age group (29).

Data obtained from the Senior Hypertension and Physical Exercise (SHAPE) study revealed that after a 6-month randomized controlled trial of aerobic and resistance training, exercisers failed to significantly reduce their SBP (30). Aortic stiffening, as measured by aorto-femoral pulse-wave velocity, failed to improve with exercise as well; this would explain the resistance of systolic hypertension to improve with physical activity in the elderly.

However, a meta-analysis involving 802 normotensive and hypertensive patients older than 50 years demonstrated a significant reduction of $2 \mathrm{mmHg}$ in SBP (20). Younger adult populations exhibit a better response to exercise. For 
instance, a study involving postmenopausal women with an average age of 55 years revealed a significant reduction in the systolic (from $142 \mathrm{mmHg}$ to $131 \mathrm{mmHg}, 8 \%$ ) and mean arterial blood pressure (from $103 \mathrm{mmHg}$ to $98 \mathrm{mmHg}, 5 \%$ ) after 24 weeks of regular walking in prehypertensive and stage I hypertensive patients. Systolic BP and mean arterial BP remained constant in the control group throughout the 24 weeks. There was no significant change in resting DBP in either group across 24 weeks. The improvement was unrelated to body composition, diet, or fasting insulin levels (31).

\section{Prescription of Exercise for Patients with Hypertension}

As a consensus, patients with hypertension should exercise most or all days of the week, for a minimum of $30 \mathrm{~min}$ of moderate aerobic exercise. Resistance training should serve as an adjunct to an aerobic-based program (32). Moderateintensity exercise should be defined as one producing an elevation to $50-60 \%$ of the maximum heart rate and associated with $40-60 \%$ of the heart rate reserve or VO2 reserve. For endurance exercise training, it appears prudent to maintain exercise blood pressure values less than 220/105 $\mathrm{mmHg}$. Moderate-intensity exercise has been proven to be as effective as vigorous activity in lowering blood pressure and is associated with fewer cardiovascular and musculoskeletal adverse effects (33). Hypertensive patients seem to maximally benefit from aerobic exercise involving large muscle groups such as walking, running, cycling, or swimming.

The prescription of exercise will vary according to the stage of hypertension, the cardiovascular status in general, and the patient's age. These factors will determine the frequency, intensity, duration, and type of exercise advised, and whether exercise testing and monitoring is required. For instance, patients who are older than 50 years with documented hypertension and suspected cardiovascular disease will need evaluation and/or monitoring by a clinical exercise physiologist and in these cases only light-to-moderate physical activity is recommended. Conversely, younger prehypertensive patients with no documented atherosclerotic disease may engage in more strenuous activities and likely do not require initial testing or monitoring 34

The evaluation of patients who will start to exercise depends on the intensity of the anticipated activity and on the patient's cardiovascular risk factors. Peak or symptom-limited exercise testing is warranted in patients with hypertension about to engage into hard physical activity or in those individuals with cardiovascular disease.

Patients without cardiovascular disease, but with blood pressure more than $180 / 110 \mathrm{mmHg}$, may benefit from exercise testing before moderate activity but not before light activity. Commonly used criteria for discontinuation of exercise testing include SBP and/or DBP values > $250 \mathrm{mmHg}$ and $115 \mathrm{mmHg}$, respectively ${ }^{(19)}$. Conversely in asymptomatic individuals with $\mathrm{BP}<180 / 110 \mathrm{mmHg}$ who are about to engage in light-to-moderate physical activity, no further testing is advised. All hypertensive patients, however, must be carefully screened with a history and physical examination for the presence of secondary causes of hypertension, the presence of risk factors, target-organ damage, and the presence of cardiovascular disease (19).

Exercise testing and monitoring is not necessary for prehypertensive or stage one or two hypertensive who are less than 50 years and have no CVD risk. Patients who are more than 50 years and have CVD or suspicion of CVD should be started with low-impact activities such as walking, cycling, and swimming but this is better to have a exercise tolerance test and interval ECG monitoring in case of chest pain.

Frequency of exercise should be 6-7 days/week with a starting intensity of 20-30 min continuous aerobic activity at comfortable pace. Then the individual is advised to maintain aerobic program at up to $85 \%$ of maximum heart rate. For patients who are overweight, weight reduction through diet modification is necessary.

\section{Conclusion}

Exercise and lifestyle modification as therapeutic intervention for the control of hypertension has proved efficacy found in many controlled studies. Aerobic exercise training can be valuable in the treatment of mild to moderate essential hypertension. ${ }^{35}$. It may cause a decrease in up to $10 \mathrm{~mm}$ $\mathrm{Hg}$ in both systolic and diastolic blood pressure. ${ }^{36}$ The blood pressure response to such exercise may also be used to screen normotensive persons for the risk of developing elevated blood pressure. ${ }^{37}$ For mild hypertension aerobic exercise and life style changes and risk factor modification such as avoidance of sedentary habit, minimizing stressful activity in office (to control white coat hypertension), weight reduction in case of obesity, dietary changes such as fatty diet and salt restriction and cessation of smoking are probably better choices. In moderate to severe essential hypertension, exercise should be combined with life style changes and medication.

\section{References}

1. Whelton PK, He J, Appel LJ. Primary Prevention of Hypertension: Clinical and Public Health Advisory from the National High Blood Pressure Education Program. JAMA. 2002; 288:1882-1888

2. Carnethon M, Gidding S, Nehgme R. Cardiorespiratory Fitness in Young Adulthood and the Development of Cardiovascular Disease Risk Factors. JAMA. 2003; 290 (23) : 3092-3100.

3. Fagard R. The Role of Exercise in Blood Pressure Control: Supportive Evidence. J Hypertens. 1995; 13:1223-1227.

4. Hagberg JM, Park JJ, Brown MD. The Role of Exercise Training in the Treatment of Hypertension: An Update. Sports Med. 2000; 30(3):193-206.

5. Pickering T. Pathophysiology of Exercise Hypertension. Herz. 1987; 12 (2):119-124. 
6. Thompson PD, Crouse SF, Goodpaster B. The Acute Versus the Chronic Response to Exercise. Med Sci Sports Exerc. 2001; 33 (6):S438-S445.

7. Shapiro L. Morphologic Consequences of Systemic Training. Cardiol Clin. 1992; 10 (2):219-226.

8. Wakatsuki T, Schlessinger J, Elson E. The Biochemical Response of the Heart to Hypertension and Exercise. Trends Biochem Sci. 2004; 29 (11): 609-617.

9. Weber K. Fibrosis and Hypertensive Heart Disease. Curr Opin Cardiol. 2000; 15:264-272.

10. De Plaen JF, Detry JM. Hemodynamic Effects of Physical Training in Established Arterial Hypertension. Acta Cardiol. 1980; 35:179-188.

11. Hagberg JM, Goldring D, Ehsani A. Effect of Exercise Training on the Blood Pressure and Hemodynamic Features of Hypertensive Adolescents. Am J Cardiol. 1983; 52:763-768.

12. Nelson L, Jennings G, Esler MD. Effect of Changing Levels of Physical Activity on Blood Pressure and Hemodynamics in Essential Hypertension. Lancet. 1986; 2:473-476.

13. Duncan JJ, Farr JF, Upton SS. The Effects of Aerobic Exercise on Plasma Catecholamines and Blood Pressure in Patients with Mild Essential Hypertension. JAMA. 1985; 254:2609-2613.

14. McAllister R, Hirai T, Musch T. Contribution of Endothelium-Derived Nitric Oxide (EDNO) to the Skeletal Muscle Blood Flow Response to Exercise. Med Sci Sports Exerc. 1995;27:1145-1151.

15. Higashi Y, Sasaki S, Sasaki N. Daily Aerobic Exercise Improves Reactive Hyperemia in Patients with Essential Hypertension. Hypertension. 1999;33:591-597.

16. Urata H, Tanabe Y, Kiyonaga A. Antihypertensive and Volume-Depleting Effects of Mild Exercise on Essential Hypertension. Hypertension. 1987;9:245-252.

17. Kinoshita A, Urata H, Tanabe Y. What Types of Hypertensives Respond Better to Mild Exercise Therapy? J Hypertens. 1998;6:S631-S633.

18. Fang J, Wylie-Rosett J, Alderman M. Exercise and Cardiovascular Outcomes by Hypertensive Status: NHANES I Epidemiologic Follow-up Study, 1971-1992. Am J Hypertens. 2005; 18:751-758.

19. American College of Sports Medicine Position Stand. Exercise and Hypertension. Med Sci Sports Exerc. 2004;36:533-553.

20. Kelly G, Sharpe K. Aerobic Exercise and Resting Blood Pressure in Older Adults: A Meta-Analytic Review of Randomized Controlled Trials. J Gerontol. 2001; 56:M298-M303.

21. Fagard R. Physical Activity in the Prevention and Treatment of Hypertension in the Obese. Med Sci Sports Exerc. 1999; 31:S624-S630.
22. PREMIER Collaborative Research Group. Effects of Comprehensive Lifestyle Modification on Blood Pressure Control. JAMA. 2003; 289 (16):2083-2093.

23. Whelton S, Chin A, Xin X. Effect of Aerobic Exercise on Blood Pressure. Ann Intern Med. 2002; 136 (7):493-503.

24. Steffen P, Sherwood A, Gullette E. Effects of Exercise and Weight Loss on Blood Pressure during Daily Life. Med Sci Sports Exerc. 2001; 33 (10):1635-1640.

25. London G, Guerin A. Influence of Arterial Pulse and Reflective Waves on Systolic Blood Pressure and Cardiac Function. J Hypertens Suppl. 1999; 17:S3-S6.

26. Antikainen R, Jousilahti P, Tuomilehto J. Systolic Blood Pressure, Isolated Systolic Hypertension and Risk of Coronary Heart Disease, Strokes, Cardiovascular Disease and All-Cause Mortality in the Middle-Aged Population. J Hypertens. 1998; 16:577-583.

27. Ferrier K, Waddell T, Gatzka C. Aerobic Exercise Training Does Not Modify Large-Artery Compliance in Isolated Systolic Hypertension. Hypertension. 2001; 38:222-226.

28. Hagberg J, Montain S, Martin W. Effect of Exercise Training in 60 to 69-Year-Old Persons with Essential Hypertension. Am J Cardiol. 1989; 64:348-353.

29. Smith S, Lovejoy J, Greenway F. Contributions of Total Body Fat, Abdominal Subcutaneous Adipose Tissue Compartments, and Visceral Adipose Tissue to the Metabolic Complications of Obesity. Metabolism. 2001; 50:425-435.

30. Stewart K, Bacher A, Turner K. Effect of Exercise on Blood Pressure in Older Persons. Arch Intern Med. 2005; 165:756-762.

31. Moreau K, Degarmo R, Langley J. Increasing Daily Walking Lowers Blood Pressure in Postmenopausal Women. Med Sci Sports Exerc. 2001;33 (11):1825-1831.

32. Pescatello L. Exercise and Hypertension: Recent Advances in Exercise Prescription. Curr Hypertens Rep. 2005; 7(4):281-286.

33. Elley CR, Aroll B. Refining the Exercise Prescription for Hypertension. Lancet. 2005; 366 (9493):1248-1249.

34. American College of Sports Medicine. Guidelines for Exercise Testing and Prescription, 6th ed. Philadelphia, PA: Lippincott, Williams \& Wilkins, 2000.

35. Levine DM, Cohen JD, Dustan HP: behavior changes and the prevention of high blood pressure. Workshop II. AHA Prevention Conference III. Behavior changes and compliance: Keys to improving cardiovascular health. Circulation 1993; 88: 1387-1390.

36. Hagberg JM, Seals DR: Exercise training and hypertension. Acta Med. Scand Suppl 1986; 711: 131-136.

37. Tanji JLChamplin JJ, Wong GY: Blood pressure recovery curves after submaximal exercise: A predictor of hypertension at ten year follow-up. Am J Hypertens 1989; 2: 135-138 